\title{
CONTRIBUIÇÕES DA TERAPIA COGNITIVO-COMPORTAMENTAL NOS CUIDADOS PALIATIVOS
}

\author{
Martha Moreira Cavalcante Castro
}

Doutora em Medicina e Saúde; Psicóloga Clínica e Hospitalar. Professora da Graduação e Pós Graduação da Escola Bahiana de Medicina e Saúde Pública

\author{
Cristina Linhares Barroso
}

Mestre em Psicologia. Professora da Graduação e Pós Graduação da Escola Bahiana de Medicina e Saúde Pública

\begin{abstract}
Resumo
O cuidado paliativo é definido como cuidar do controle efetivo da dor e de outros sintomas, presentes em pessoas que têm doenças graves sem possibilidade de cura. A dor, enquanto sintoma físico e o sofrimento resultante da ameaça de perda do eu, imposta pela doença. Esta se relaciona à sua história de vida, às suas crenças e medos: da solidão, do desconhecido, da separação de quem se ama, de interromper planos e sonhos traçados ao longo da vida. Tantos outros medos se apresentam no curso do adoecimento, favorecem respostas de tensão e dificuldades de manter qualidade na vida. A experiência do adoecimento é muito difícil não só para o paciente, mas também para seus familiares, amigos, cuidadores e equipe de saúde envolvida em seu tratamento. Devido à complexidade do cuidado dos pacientes e familiares, é necessária uma equipe multiprofissional para o adequado controle dos sintomas. A Terapia Cognitivo-Comportamental tem se mostrado um instrumento bastante eficaz no tratamento desses doentes, com a utilização de técnicas de avaliação dos aspectos sensitivos, afetivos e comportamentais; escalas de avaliação da dor e incapacidade; atitudes encorajadoras; psicoeducação e estratégias de alívio, de tensão e ansiedade que são treinadas com os sujeitos atendidos e seus familiares. É no alívio de quadros álgicos intensos e no acolhimento do sofrimento do paciente e sua família que consiste a resignificação e redirecionamento da sua vida apesar da doença.
\end{abstract}

Palavras-chave: Cuidado Paliativo; Terapia Cognitivo-Comportamental; Tratamento.

\section{CONTRIBUTIONS OF COGNITIVE-BEHAVIORAL THERAPY IN PALLIATIVE CARE}

\begin{abstract}
Palliative care is defined as care for the effective control of pain and other symptoms present in people who have serious illnesses with no cure. The pain, while physical symptom, is the suffering resulting from the threat of loss of self, imposed by the disease. This relates to their life history, their beliefs and fears: the loneliness, the unknown, the separation of one you love, interrupting plans and dreams traced throughout life. So many other fears arise in the course of illness, promoting stress responses and difficulties of maintaining quality of life. The experience of illness is very difficult not only for the patient but also for their families, friends, caregivers and health professionals involved in the care. Due to the complexity of care for patients and families, a multidisciplinary team is necessary for adequate control of symptoms. The Cognitive-Behavioral Therapy has been shown to be a highly effective instrument in the treatment of these patients with the use of valuation techniques of sensitive aspects, affective and behavioral; rating scales of pain and disability; encouraging attitudes; psychoeducation and strategies of relief, tension and anxiety that are trained with the subjects attended and their families. It is in the relief of intense pain conditions and in the welcoming the suffering of the patient and his family that lies a new meaning and redirection of his life despite the disease.
\end{abstract}

Keywords: Palliative Care; Cognitive-Behavioral Therapy; Treatment. 
O conceito de Cuidados Paliativos originou-se no movimento hospice, organizado por Cecily Saunders e seus colaboradores, cuja filosofia é o cuidar a partir do controle efetivo da dor e de outros sintomas, presente em fases avançadas das doenças, e o cuidado com as dimensões psicológicas, sociais e espirituais de pacientes e familiares. ${ }^{(1)}$

A palavra hospice se origina do latim hospes, que significa estranho e depois anfitrião; hospitalis significa amigável, ou seja, boas-vindas ao estranho, e evolui para o significado de hospitalidade. O termo palliare também se origina do latim e significa proteger, amparar, cobrir, abrigar. A perspectiva do cuidar e não somente curar surge de forma ampla como foco principal. $^{(2)}$

Doentes em cuidados paliativos apresentam alta ocorrência de sintomas físicos e psíquicos. Dor, por exemplo, alteração cognitiva, constipação intestinal e fadiga, são os mais frequentes.

Dor e sofrimento são conceitos com elementos comuns, mas não são a mesma coisa. Dor é uma vivência sensorial e emocional desagradável frente a um estímulo, geralmente uma lesão, com repercussões físicas, sociais e espirituais. Sofrimento resulta da ameaça de ruptura do eu frente a situações-limite como dor, perdas, morte, quebra de valores etc. O sofrimento está relacionado à subjetividade do sujeito, aos valores adquiridos ao longo da vida, traduz o temor do incontrolável e reflete a fragilidade e vulnerabilidade do ser humano.

O sofrimento, gerado pelo temor da doença desde o seu diagnóstico, perpassa o tratamento e se agrava com a possibilidade da morte. Se morrer é fato para todos os seres vivos, como humanos, o que nos diferencia dos demais é a consciência da nossa finitude e a resposta a isso, em geral, é medo e sofrimento. O sofrimento pode conter o medo da solidão, do desconhecido, da separação de quem amamos, de interromper planos e sonhos traçados ao longo da vida e tantos outros medos que se apresentam no curso do adoecimento. ${ }^{(3)}$

De modo geral, as pessoas não se preparam para adoecer, pois a crença dominante é de vida plena, longa, satisfatória e, acima de tudo, saudável; ficar doente, não faz parte do "script". $\mathrm{O}$ adoecimento exige uma série de movimentos internos e externos: definir passos do tratamento, autoperceber-se na nova condição, ajustar as expectativas pessoais e de seus familiares sobre 
tratamento e recuperação, mudanças no estilo e na qualidade de vida e tantas outras demandas exigirão do indivíduo grande investimento de energia.

A experiência do adoecimento é muito difícil não só para o paciente, mas também para seus familiares, amigos, cuidadores e equipe de saúde envolvida em seu tratamento. O sistema familiar funciona de acordo com regras e padrões próprios e o adoecimento de um membro “desestabiliza" esse sistema, que precisará ser reorganizado para atender às demandas advindas da doença e tratamento. As adaptações feitas pelos membros da família, mudanças de papéis, flexibilidade e cuidado integral proporcionarão alívio do sofrimento. ${ }^{(4)}$ Adaptação não significa resolução, no sentido da aceitação completa e definitiva das perdas, mudanças etc., ao contrário, envolve a descoberta de maneiras de seguir em frente com a vida. A família promove a adaptação e se torna novamente um sistema funcional pelo reconhecimento, compartilhado por todos os membros da família, da realidade da dor e da morte, fazendo disso uma experiência comum de perda. A comunicação entre a família e os profissionais de saúde pode auxiliar na reorganização do sistema familiar e no reinvestimento desses em outras relações e projetos de vida. ${ }^{(5)}$

É importante compreender o significado que os pacientes dão à experiência de dor como fator de adaptação. Para isso, é relevante ressaltar qual o papel das crenças para o paciente com dor, assim como a influência na sua percepção final da dor e nas estratégias de enfrentamento usadas por ele. As crenças podem envolver conceitos próprios sobre o que é dor, seu significado e a compreensão pessoal da experiência dolorosa. ${ }^{(6)}$

Do mesmo modo, quando o indivíduo acredita que pode ter controle sobre sua vida, percebe menos a intensidade da dor, mostra maiores níveis de atividade e utiliza estratégias de enfrentamento mais adaptativas, o que reflete em um maior nível de adaptação. Aquele que se vê indefeso ante a impossibilidade de controlar sua dor, ao contrário, apresenta aumento da frequência e da intensidade dos episódios dolorosos. ${ }^{(7-9)}$

O preparo dos doentes e cuidadores para o uso de qualquer método para o controle da dor deve ser feito de modo continuado. A ação educativa visa torná-los agentes de autocuidado e participantes conscientes do processo terapêutico. Como apreciar a dor envolve aspectos emocionais e cognitivos, o que o doente sabe acerca da sua dor e as crenças que tem sobre ela, vão definir a forma como ele lida com a situação. Logo, educar pacientes e cuidadores é a chave 
fundamental para o efetivo controle da dor. Além da educação continuada do processo álgico, o uso da Terapia Cognitivo-Comportamental (TCC) tem-se mostrado um instrumento bastante eficaz no tratamento desses doentes.

Como na TCC os processos cognitivos dizem respeito à causa dos comportamentos disfuncionais, distorções cognitivas frente às diversas possibilidades de interpretação da realidade podem comprometer a saúde biopsicossocial do indivíduo. O paciente com dor, além de apresentar fisiopatologia específica para seu quadro álgico, ainda é vulnerável a estímulos ambientais, os quais geram comportamentos e cognições disfuncionais. Isso faz com que o estímulo e a resposta do processamento cognitivo seletivo falhem na realidade pessoal do indivíduo, o que contribui para a manutenção de seu quadro álgico. ${ }^{(10)}$

Quando o tratamento evolui satisfatoriamente, ocorre maior flexibilidade nas crenças que permeiam os comportamentos dolorosos e a diminuição da ativação dos pensamentos automáticos negativos, - o que favorece o aumento de pensamentos mais funcionais. Consequentemente, o paciente torna-se mais ativo no tratamento e passa a ter maior habilidade para funcionar em presença da dor. ${ }^{(11)}$

A TCC no tratamento da dor busca auxiliar os pacientes a se tornarem capazes de avaliar o impacto que pensamentos e sentimentos negativos de dor provocam na manutenção de comportamentos inadequados, encorajando-os a conservarem a orientação para solucionar problemas e a desenvolverem recursos para aprenderem a lidar com a dor. Dessa forma, os pacientes são incentivados a reconhecer as conexões nas respostas de cognição, humor e comportamento, juntamente com suas consequências e, finalmente, são encorajados a expandir os ganhos da clínica para além dela. ${ }^{(12)}$

Para o controle álgico, intervenções não farmacológicas como apoio emocional, métodos físicos, educacionais e cognitivo-comportamentais são fundamentais para o adequado manejo da dor.

Além da avaliação geral do paciente, algumas técnicas cognitivo-comportamentais estão incluídas no tratamento do indivíduo em Cuidados Paliativos tais como: Técnica de Psicoeducação; Técnica de Treino Assertivo e de Habilidade Social; Reestruturação Cognitiva e Técnicas de aquisição de estratégias para alívio dos estados de tensão e ansiedade. 
Avaliação dos aspectos sensitivos, afetivos e comportamentais devido à frequente presença de sintomas ansiosos e depressivos; uso de escalas de avaliação da dor e da incapacidade que favorecem o planejamento terapêutico; atitudes encorajadoras e a exposição da proposta terapêutica.

A Psicoeducação sobre dor e seu controle, de análise e reorganização do comportamento e do pensamento (trabalhos individuais sobre atitudes e crenças frente à dor);

A Técnica de Treino Assertivo estimula o paciente a expressar seus sentimentos negativos ou positivos com domínio pessoal e a Técnica de Habilidade Social encoraja o paciente a participar (sempre que possivel) de atividades sociais (passeio em shopping, ida a igrejas, aniversários de amigos, encontro de família) mesmo com suas limitações.

A Reestruturação Cognitiva permite que o paciente identifique pensamentos que afetam seu humor e estado físico, permitindo a correção dos erros lógicos ou de conteúdo, assim como rever crenças disfuncionais acerca do seu adoecimento. ${ }^{(13)}$

As técnicas de aquisição de estratégias para alívio dos estados de tensão e ansiedade (técnicas de relaxamento, de distração e de imaginação dirigida, entre outras), podem ser ensinadas aos doentes e cuidadores para que estes a desenvolvam no domicílio.

O relaxamento pode ser definido como o estado de relativa ausência de ansiedade e tensão muscular; a serenidade da mente e dos músculos. As técnicas que utilizam a respiração como estratégia para o relaxamento tentam tornar o indivíduo consciente do processo de respiração. Quando a atenção é focada na respiração, é removida das áreas de tensão. Essas técnicas são muito flexíveis, fáceis de serem aprendidas e utilizadas. As técnicas de relaxamento muscular progressivo são desenvolvidas a partir de procedimento estruturado de contração e relaxamento de grupos musculares específicos e em sequência pré-estabelecida. Auxiliam o indivíduo a ficar mais consciente dos locais de tensão muscular e despendem cerca de 20 minutos para a realização de toda a sequência. $\mathrm{O}$ autorrelaxamento é muito difundido entre as pessoas e consiste no uso de frases afirmativas e agradáveis, que o indivíduo repete para si, como por exemplo, "minha mente está calma", "meus músculos estão relaxados", entre outras.

As técnicas de distração para o controle da dor podem ser compreendidas como a focalização da atenção em outro estímulo que não a dor. Aumenta-se a estimulação de outros 
canais sensoriais como a audição, a visão e o tato, e atenção à dor fica prejudicada pela atenção a outro estímulo. O estímulo pode ser externo (ouvir música por fone de ouvido, assistir filme) ou interno (cantar ou fazer contas mentalmente). O uso da distração pode aumentar a tolerância à dor e a sensação de controle. Ouvir música, jogar jogos, utilizar técnicas de respiração, assistir filmes, ler, fazer contas ou cantar mentalmente, marcar o compasso de uma música com os pés ou com as mãos, imaginar cenas agradáveis, são exemplos de técnicas de distração que podem ser utilizadas.

A imaginação dirigida envolve o uso de imagens mentais e é frequentemente referida como a criação de cenas agradáveis, visualização, sonhar acordado ou fantasiar. Fazer uma viagem imaginária à praia ou ao campo são recursos muito utilizados. O objetivo é produzir relaxamento, experimentar sensação de bem-estar, retirar o foco e diminuir a percepção da dor.

À equipe de saúde cabe orientar pacientes, familiares e cuidadores na atenção aos sintomas, garantir a realização do tratamento visando seu bem-estar físico e psicológico e promover aliança terapêutica, a fim de alimentar a mútua confiança. Acolher o sofrimento do paciente, buscando fortalecer sua identidade, autoestima e autonomia, são de extrema importância para que o doente possa ressignificar e redirecionar sua vida, apesar da doença. Familiares e cuidadores devem ser ouvidos ativamente, visando a identificar oportunidades de mudanças que melhorem o bem-estar geral, a busca de recursos adaptativos e o fortalecimento das inter-relações em todo o sistema.

A comunicação entre profissional de saúde e paciente é tida como ponto importante a ser trabalhado na equipe, principalmente pelo psicólogo, pois é seu dever informar, esclarecer, visto que há o temor pelo desconhecido e a informação transmitida adequadamente pode reduzir sintomas ansiosos e depressivos. O apoio psicológico reduz os dias de internação, complicações e uso de analgésicos pós-cirúrgicos. É tarefa do psicólogo ainda auxiliar o paciente quanto à aceitação do seu diagnóstico e/ou prognóstico da doença. ${ }^{(14)}$

Problemas de comunicação na relação paciente/profissional de saúde em geral, podem dificultar a adesão do paciente ao tratamento. ${ }^{(15)}$ 
O psicólogo pode auxiliar os membros da família a permanecer unidos, proporcionar espaços para falar sobre a morte, sobre seus sentimentos, pois a falta de expressão destes pode gerar sentimentos de culpa e raiva. ${ }^{(16)}$

A fim de preparar o aluno de psicologia para lidar com pessoas acometidas pelo câncer, a Escola Bahiana de Medicina e Saúde Pública fez uma parceria com o Grupo de Apoio à Criança com Câncer (GACC), cujo objetivo era desenvolver uma avaliação do paciente oncológico infantil diante do seu adoecer, da morte e as repercussões advindas do seu tratamento; avaliar as relações com a família e equipe de saúde e, dessa forma, favorecer a essas pessoas um maior enfrentamento do seu adoecimento. Essas crianças eram assistidas e cuidadas pelos alunos durante todo o percurso do seu tratamento, desde a avaliação psicológica até o acompanhamento psicoterápico. Essa experiência revelou que, o estudante de psicologia, na ênfase da saúde, ao se deparar com temas como a psiconcologia geral e infantil, ao vivenciar os aspectos psicológicos do paciente com câncer; o suporte psíquico à família do paciente oncológico, os tipos de tratamento, relação equipe de cuidadores $\mathrm{X}$ paciente e os aspectos gerais da morte e do morrer, mostrou-se preparado para os desafios da prática do psicólogo no atendimento às pessoas na fase final da doença.

\section{REFERÊNCIAS}

1. Pimenta CAM, Mota DDCF, da Cruz DALM. Dor e cuidados paliativos: enfermagem, medicina e psicologia. São Paulo: Manole; 2006. Cap. 2, Cuidados paliativos: Conceitos básicos, histórico e realizações da Associação Brasileira de Cuidados Paliativos e da Associação internacional de Hospice e Cuidados Paliativos; p.16-28.

2. Figueiredo MGMCA, Figueiredo MTA. Cuidados paliativos. In: Incontri D, Santos FS, organizadores. A arte de morrer: visões plurais. São Paulo: Comenius; 2007; p. 196-206.

3. Borges ADVS, da Silva EF, Toniollo PB, Mazer SM, do Valle ERM, dos Santos MA. Percepção da morte pelo paciente oncológico ao longo do desenvolvimento. Psicol Est. 2006;11:361-9.

4. Walsh F, McGoldrick M. Morte na família: sobrevivendo às perdas. Porto Alegre: Artmed,1998. 
5. Miceli AVP. Dor crônica e subjetividade em oncologia. Rev Bras Cancerol. 2002;48:363-73.

6. Araújo CF, Shinohara, H. Avaliação e diagnóstico em terapia cognitivo-comportamental. Inter Psicol. 2002;6:37-43.

7. Basler HD, Jäkle C, Kröner-Herwig B. Incorporation of cognitive-behavioral treatment into the medical care of chronic low back patients: a controlled randomized study in german pain treatment centers. Patient Educ Couns. 1997;31:113-24.

8. Ortiz MTA, Martel LC. Importancia de las creencias en la modulación del dolor crônico: concepto y evaluación. Apuntes Psicol. 2001;19(3):453-70.

9. Brox JI, Sorensen R, Friis A, Nygaard O, Indahl A, Keller A, et al. Randomized clinical trial of lumbar instrumentted fusion and cognitive intervention and exercises in patients with chronic low back pain and disc degeneration. Spine 2003;28:1913-21.

10. Williams DA, Cary MA, Groner KH, Chaplin W, Glazer LJ, Rodriguez AM, et al. Improving physical functional status in patients with fibromyalgia: a brief cognitive behavioral intervention. J Rheumathol. 2002;29:1280-86.

11. Burns JW, Kubilus A, Bruhel S, Harden RN, Lofland K. Do changes in cognitive factors influence outcome following multidisciplinary treatment for chronis pain?: a cross-lagged panel analysis. J Consult Clin Psychol. 2003;71:81-91.

12. Hiller W, Heuser J, Fichter M. The DSM IV nosology of chronic pain: a comparison of pain disorder and multiple somatization syndrome. Eur J Pain 2000;4:45-55.

13. Sudak DM. Terapia cognitivo-comportamental na prática, São Paulo: Artmed; 2008.

14. Gorayeb R. A prática da psicologia hospitalar. In: Marinho MK, Caballo VE, organizadores. Psicologia clínica e da saúde. Londrina: UEL; 2001. p. 263-78.

15. Gorayeb R, Guerrelhas F. Sistematização da prática psicológica em ambientes médicos. Rev Bras Ter Comp Cognit 2003;5:11-9.

16. Labate RC, de Barros CG. Uma possibilidade de escuta a uma família enlutada: ressignificando a experiência de perda. Rev SPAGESP 2006;7:35-40. 